\title{
Re-educating tumour-associated macrophages with nanoparticles
}

Tumour-associated macrophages (TAMs) are thought to have an immune-suppressive function and therefore promote tumour survival. An article in Nature Biomedical Engineering describes nanoparticles that contain a dual agonist of the innate immune receptors toll-like receptor 7 (TLR7) and TLR8 that are selectively taken up by TAMs and alter their phenotype such that they promote, rather than hinder, immune surveillance in mouse models.

The authors began with a cell morphology-based screen to identify small molecules that can change macrophages from having a pro-repair, immune-inhibitory, M2-like phenotype to one that is inflammatory and M1-like. M1-like macrophages recruit cytotoxic $T$ cells to promote immune surveillance. A panel of 38 drugs curated from the literature was screened, and agonists that affected TLR7 and/or TLR8 produced the largest effects on cell polarization. Of these, R848, a dual TLR7 and TLR8 agonist, had the most potent effects on cell morphology, and nanomolar concentrations combining

CDNP-R848

treatment with blockade of [PD1] was synergistic 5

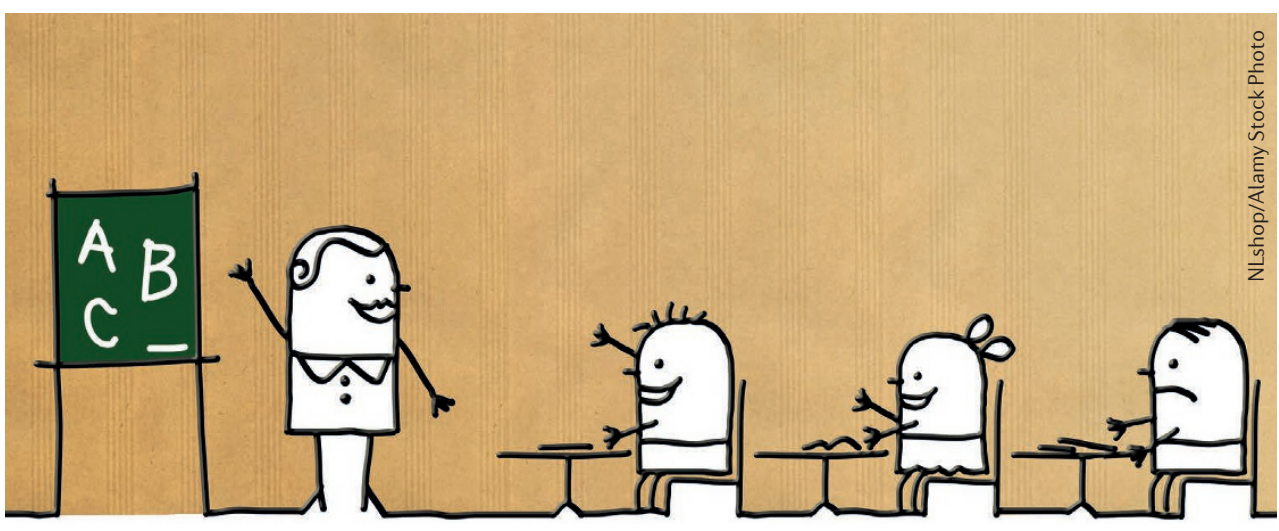

R848-containing CDNPs (CDNP-R848) similarly localized to tumours in an orthotopic lung adenocarcinoma model. In the MC38 model, this localization was associated with increased IL-12 production from TAMs.

Treatment of mice bearing MC38 tumours with CDNP-R848 reduced tumour growth and improved survival relative to treatment with CDNP or R848 alone. As these TAMs were now immune-stimulating, the authors reasoned that CDNP-R848 could improve the efficacy of immune checkpoint blockade. Indeed, combining CDNP-R848 treatment with blockade of programmed cell death protein 1 (PD1) was synergistic: dual treatment resulted in tumour shrinkage, and, in two of seven tumours, complete tumour regression. Those two mice were resistant to secondary tumour challenge, suggesting that the immune cells in these mice retained anti-tumour memory.

CDNP-R848 was also able to sensitize the B16.F10 melanoma model, which is resistant to treatment with anti-PD1 agents, to immune checkpoint blockade. CDNP-R848 reduced tumour growth and prolonged survival in this model, and had synergistic effects with anti-PD1 therapy.

Harnessing TAMs to direct immune cells to the tumour is an attractive proposition. This article proves the principle that re-educating TAMs to have an M1-like phenotype can promote tumour regression and increase the efficacy of immune checkpoint blockade, and provides the tools with which to do it.

Megan Cully

ORIGINAL ARTICLE Rodell, C. B. et al. TLR7/8-agonist-loaded nanoparticles promote the polarization of tumour-associated macrophages to enhance cancer immunotherapy. Nat. Biomed. Eng. https://doi.org//10.1038/s41551-018-0236-8 (2018) FURTHER READING Jiang, W. et al. Lessons from immuno-oncology: a new era for cancer nanomedicine? Nat. Rev. Drug Discov. 16, 369-370 (2017) 